\title{
Reverse bias-dependence of schottky barrier height on silicon carbide: influence of the temperature and donor concentration
}

\author{
Abdelhakim Latreche \\ Département des Sciences de la Matière, Université de Bordj Bou Arreridj, 34000, Algeria \\ E-mail: hlat26@yahoo.fr
}

Copyright $\odot 2014$ Abdelhakim Latreche. This is an open access article distributed under the Creative Commons Attribution License, which permits unrestricted use, distribution, and reproduction in any medium, provided the original work is properly cited.

\begin{abstract}
The work deals with the dependences of the Schottky barrier height $(\mathrm{SBH})$ on the reverse bias voltage, temperature and on donor concentration of metal/4H-SiC Schottky diodes. Using the tunneling modeling we have shown that the Schottky barrier height on silicon carbide strongly depends on the reverse bias voltage, temperature and doping concentration. At room temperature, the Schottky barrier height increases with increasing the reverse bias voltage at high doping concentration (about $10^{16} \mathrm{~cm}^{-3}$ ), while, at low doping concentration (about $10^{15} \mathrm{~cm}^{-3}$ ) the Schottky barrier height decreases with increasing the reverse bias voltage. These behaviors are independent of the Schottky barrier lowering effect. That means other effects occur at the barrier and depend on the reverse applied bias. The barrier height increases with increasing temperature and doping concentration under reverse bias conditions. The barrier heights extracted from the Padovani-Stratton formulas are close to the barrier heights extracted from the Tsu-Esaki formula in particular for the thermionic-field emission.
\end{abstract}

Keywords: Extraction, Schottky Barrier Height, Reverse Bias, Tunneling, Silicon Carbide.

\section{Introduction}

Silicon carbide is currently of great interest in high voltage power devices. Its properties of high critical field strength, reasonable carrier mobilities, wide band gap, and high thermal conductivity make it a useful material for high frequency, high temperature, and high power devices [1], [2]. However, due to the high electric fields normally encountered in SiC devices, the reverse leakage current of Schottky diodes can be significantly enhanced prior to junction breakdown due to the tunneling mechanism [3]. The high electric fields encountered in SiC and other wide band gap materials lead to significant tunneling through the Schottky barrier thus increasing the leakage current by many orders of magnitude over that predicted by simple thermionic emission (TE) calculations [3-10]. However, the tunneling leakage current, which calculated with the extracted SBH from forward I-V data, is discrepancy of the experimental data [3], [4], [9]. In these works, the authors assume that the barrier height does not depend on the applied bias despite the bias voltage is elevated. According to Rhoderick [11], there are several reasons why the Schottky barrier height $\left(\phi_{b}\right)$ may depend on the applied bias such as interfacial layer and effect of the image force. These both factors reduce the barrier height by an amount that depends on the electric field in the semiconductor, and hence on the applied bias. Rhoderick [11] supposed that $\phi_{b}$ depends linearly on the applied bias V, which is true for small values of forward bias voltage $(\mathrm{V})$, so, the barrier height can be written as.

$\phi_{b}=\phi_{b 0}(V=0)+\beta V$

Where $\phi_{b 0}(V=0)$ is the barrier height at $V=0$ volts, $\beta$ is the slope of the straight line.

Until the present no one proved experimentally if it is a wrong or a true hypothesis weather in the forward bias or in the reverse bias. The influence of the applied voltage on the $S B H$ in the case of forward bias conditions where the predominant mechanism is the thermionic emission does not appear because the magnitude of applied voltage is very small (about $1 \mathrm{~V}$ ). On the contrary, the magnitude of the reverse applied voltage is very high and thus we can predict that the $S B H$ can be influenced by the applied bias. In this paper, we will study the reverse bias dependence of the 
Schottky barrier height on Silicon carbide and show the influences of the temperature and donor concentration on the $S B H$ under reverse bias conditions. For this purpose we assume the domination of tunneling current for the whole reverse bias, temperature and doping level ranges and we use both models of tunneling: Tsu-Esaki's [12] and PadovaniStratton's [13] formulas for extracting the $S B H$ from reverse $I-V$ measurements studied in the literature.

Padovani and Stratton [13] analyzed tunneling currents in Schottky barriers from the standpoint of the field and thermionic-field emission using a one dimensional Wentzel-Kramer-Brillouin (WKB) approximation. However, their analysis ignored image force effects and used a simple parabolic barrier shape.

The field emission current density in the reverse bias applied is expressed by the following equation [13]

$$
J_{F E}=\frac{A^{*} T^{2} \pi E_{00} \exp \left[-2 q \phi_{b}^{3 / 2} / 3 E_{00}\left(\phi_{b}-V\right)^{1 / 2}\right]}{k_{B} T\left[\phi_{b} /\left(\phi_{b}-V\right)\right]^{1 / 2} \sin \left\{\pi k_{B} T\left[\phi_{b} /\left(\phi_{b}-V\right)\right]^{1 / 2} / E_{00}\right\}}
$$

Where $\mathrm{E}_{00}$ is a constant related to the $W K B$ expression for the transmission of the barrier and is given as follows

$$
E_{00}=\frac{q \mathrm{~h}}{2} \sqrt{\frac{N_{D}}{m_{e}^{*} \varepsilon_{S}}}
$$

Where $N_{D}$ is the semiconductor ( $n$-type) doping concentration, $\varepsilon_{S}$ is the semiconductor permittivity, $A *$ is the effective Richardson constant, theoretically equal to $146 \mathrm{~A} / \mathrm{K}^{2} \mathrm{~cm}^{2}[14]$

The thermionic-field emission current density in the reverse bias applied is expressed by the following equation [13]

$$
J_{T F E}=\frac{A^{*} T}{k_{B}} \sqrt{\pi E_{00 q}\left(-V+\frac{\phi_{b}}{\cosh ^{2}\left(E_{00} / k_{B} T\right)}\right)} \exp \left(\frac{-q \phi_{b}}{E_{0}}\right) \exp \left(-\frac{q V}{\varepsilon^{\prime}}\right)
$$

Where

$$
\begin{aligned}
& \varepsilon^{\prime}=\frac{E_{00}}{\left(E_{00} / k_{B} T\right)-\tanh \left(E_{00} / k_{B} T\right)} \\
& E_{0}=E_{00} \operatorname{coth}\left(\frac{E_{00}}{k_{B} T}\right)
\end{aligned}
$$

Field emission occurs for $\mathrm{c}_{1}>k_{B} T$ and thermionic field emission for $\mathrm{c}_{1}<k_{B} T$ where $\mathrm{c}_{1}$ is given by

$$
c_{1}=\left\{E_{00}{ }^{-1}\left[\phi_{b} /\left(\phi_{b}-V\right)\right]^{1 / 2}+\left(-0.5 q E_{00} V\right)^{1 / 2}\right\}^{-1}
$$

The Tsu-Esaki expression is the most prominent and almost exclusively used expression to describe tunneling transitions. The reverse current density is given by [3], [7], [12], and [15]

$J_{T} \& E=\frac{A^{*} T}{k_{B}} \int_{0}^{\infty} T(E) \ln \left(\frac{1+\exp (-q \zeta-E) / k_{B} T}{1+\exp (-q \zeta-q V-E) / k_{B} T}\right) d E$

Where $\zeta$ is the difference between the conduction band and the equilibrium Fermi level, $E$ is the electron transversal energy component. $T(E)$ is the transmission coefficient. In this work, we use the $W K B$ approximation because it offers an analytical solution and it is valid for the Schottky barrier diodes [16].

\section{Extraction method of the barrier height using the reverse I-V characteristics}

For extracting the barrier height $\left(\phi_{b}\right)$ from the reverse $I-V$ characteristic, we assume that the tunneling process is the dominant mechanism. First, we assume that the $\phi_{b}$ is independent of the applied bias, and then we extract the values of $\phi_{b}$ and the effective mass $\left(\mathrm{m}^{*}\right)$ which verified the best fit of the experimental data. For that, we use the criterion (S) which presents the sum of the squares of the relative differences between the measured and the ideal values. This criterion was proposed for the first time by Osvald [17] for extracting the parameters of Schottky diode from the forward $I-V$ characteristic.

$S=\sum_{i=1}^{N}\left(\frac{I_{i}^{T h}-I_{i}^{\exp }}{I_{i}^{T h}}\right)^{2}$

Where $I_{i}^{\exp }$ is the ith experimental value, $I_{i}^{T h}$ is the fitting value of the current, i.e. given by the $E q$. (8) for $\mathrm{V}=\mathrm{V}_{\mathrm{i}}$, and $N$ is the number of measuring points.

Second, we assume that the $\phi_{b}$ is dependent on the applied bias and use the theoretical value of the effective mass for $4 \mathrm{H}-\mathrm{SiC}\left(m^{*} \approx 0.2 m\right)$ [18-20]. So, we can use the Tsu-Esaki formula or the Padovani-Stratton formulas for extracting the $\mathrm{SBH}$. For that, we can solve numerically the following equations by Newton's method. 
$J_{T} \& E\left(V_{i}\right)=J_{\exp }^{i}\left(V_{i}\right)$

$J_{T F E}\left(V_{i}\right)=J_{\exp }^{i}\left(V_{i}\right)$

$J_{F E}\left(V_{i}\right)=J_{\exp }^{i}\left(V_{i}\right)$

Where $J_{\exp }^{i}\left(V_{i}\right)$, is the reverse current density for each bias voltage measurement. The Eq. (10) uses the Tsu-Esaki formula and the Eqs. (11) And (12) use the Padovani-Stratton formulas.

\section{Results and discussion}

In this study we use the data on silicon carbide $(4 \mathrm{H}-\mathrm{SiC})$ previously published by several authors. The wafers had an $n$ type epitaxial layer. The type of diode, doping concentration $N_{D}$, temperature and the forward barrier height for each diode are summarized in the Table 1. As shown in this Table all the semiconductors used in this study are lightly doped and the doping level is varied from $9 \times 10^{14} \mathrm{~cm}^{-3}$ to $1.6 \times 10^{16} \mathrm{~cm}^{-3}$.

Table 1: Properties and Characterization of the Diodes Collected from Several Authors.

\begin{tabular}{llllll}
\hline Type & $\mathrm{N}_{\mathrm{D}}\left(\mathrm{cm}^{-3}\right)$ & $\mathrm{T}(\mathrm{K})$ & $\phi_{b}^{I-V}(V)$ & Reference & Observations \\
\hline $\mathrm{Ti} / 4 \mathrm{H}-\mathrm{SiC}_{\text {ref } 4}$ & $10^{16}$ & 300 & 1.21 & Furno [4] & Junction termination extension \\
$\mathrm{Ni} / 4 \mathrm{H}-\mathrm{SiC}_{\text {ref } 5}$ & $1.6 \times 10^{16}, 3.5 \times 10^{15}$ & 293 & $1.3,1.5$ & Schoen [5] & Boron implant edge termination \\
$\mathrm{Ti} / 4 \mathrm{H}-\mathrm{SiC}_{\text {ref 5 }}$ & $3.5 \times 10^{15}$ & 293 & 0.85 & Schoen [5] & Boron implant edge termination \\
$\mathrm{Ti} / 4 \mathrm{H}-\mathrm{SiC}_{\text {ref 5 }}$ & $1.6 \times 10^{16}$ & $293-528$ & $0.8-?$ & Schoen [5] & Boron implant edge termination \\
$\mathrm{Ti}(\mathrm{Ni}) / 4 \mathrm{H}_{-}-\mathrm{SiC}_{\text {ref } 6}$ & $3 \times 10^{15}$ & $301-517$ & $1.4-1.47$ & Vassilevski [6] & Junction termination extension \\
$\mathrm{Ti} / 4 \mathrm{H}-\mathrm{SiC}_{\text {ref 18 }}$ & $7 \times 10^{15}$ & $297-423$ & 1.16 & Itoh [18] & Boron implant edge termination \\
$\mathrm{Ni} / 4 \mathrm{H}-\mathrm{SiC}_{\text {ref 21 }}$ & $6.1 \times 10^{15}$ & $300-573$ & $1.38-1.37$ & saxena [21] & Field oxide \\
$\mathrm{Ni} / 4 \mathrm{H}-\mathrm{SiC}_{\text {ref 22 }}$ & $2 \times 10^{15}$ & 300 & - & Nigam [22] & JTE, circular diode, area 0.04mm ${ }^{2}$ \\
$\mathrm{Ti} / 4 \mathrm{H}-\mathrm{SiC}_{\text {ref 23 }}$ & $2 \times 10^{15}-7.4 \times 10^{15}$ & 300 & $1.2-1.17$ & Ohtsuka [23] & No edge termination \\
$\mathrm{Ni} / 4 \mathrm{H}-\mathrm{SiC}_{\text {ref 24 }}$ & $2 \times 10^{15}$ & $359-522$ & 1.53 & Ivanov [24] & guard rings: planar $p-n$ junctions \\
$\mathrm{Ni} / 4 \mathrm{H}-\mathrm{SiC}_{\text {ref 25 }}$ & $9 \times 10^{14}$ & $361-470$ & 1.12 & Ivanov [25] & protective $p$-type rings \\
\hline
\end{tabular}

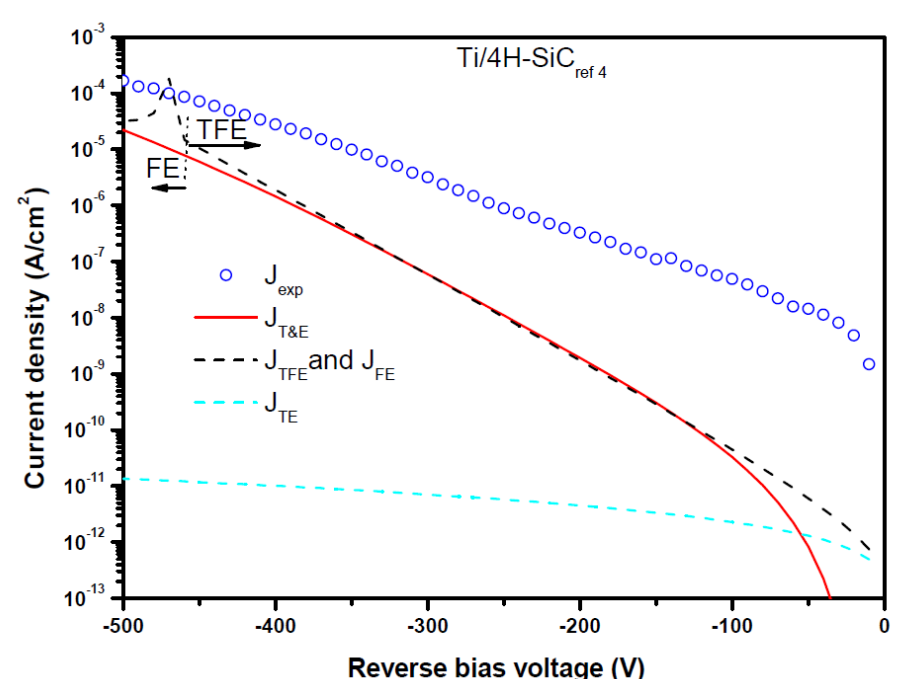

Fig. 1: Comparison of Calculating Current Densities (Thermionic Emission, Field Emission, Thermionic Field Emission and Tsu-Esaki's Expression Calculated With $\phi_{b}^{I-V}=1.21 \mathrm{~V}$ ) and Experimental Leakage Current For Ti/4H-SiC ${ }_{\text {ref } 4}$ Schottky Diode. the Experimental Results are From Furno [4].

Fig. 1 shows a comparison of calculating reverse current densities with experimental result obtained for $\mathrm{Ti} / 4 \mathrm{H}-\mathrm{SiC}_{\text {ref }} 4$ $\mathrm{SD}$. The calculated reverse current densities are obtained with the extracted barrier height $\phi_{b}^{I-V}=1.21 \mathrm{~V}$ from forward I-V measurements. The present result clearly shows the importance of tunneling currents during reverse bias operation of Schottky diodes in high field materials such as SiC. In contrast, and as shown in Fig. 1, it may be noted that thermionic emission theory predicts much smaller voltage dependence. The current densities obtained by the PadovaniStratton formulas are close to the current density obtained by the Tsu-Esaki formula in particular for the Thermionic field emission. The transition between field and thermionic field emission occurs at approximately - $460 \mathrm{~V}$. However, Fig. 1 shows a strong difference between the slope of the $\ln (\mathrm{I})-\mathrm{V}$ plots obtained by the tunneling numerical methods and 
the slope of the experimental $\ln (\mathrm{I})-\mathrm{V}$ plot. The electron effective mass $m^{*}$ is the parameter determining the slope of the calculated reverse characteristics as shown in Fig. 2.

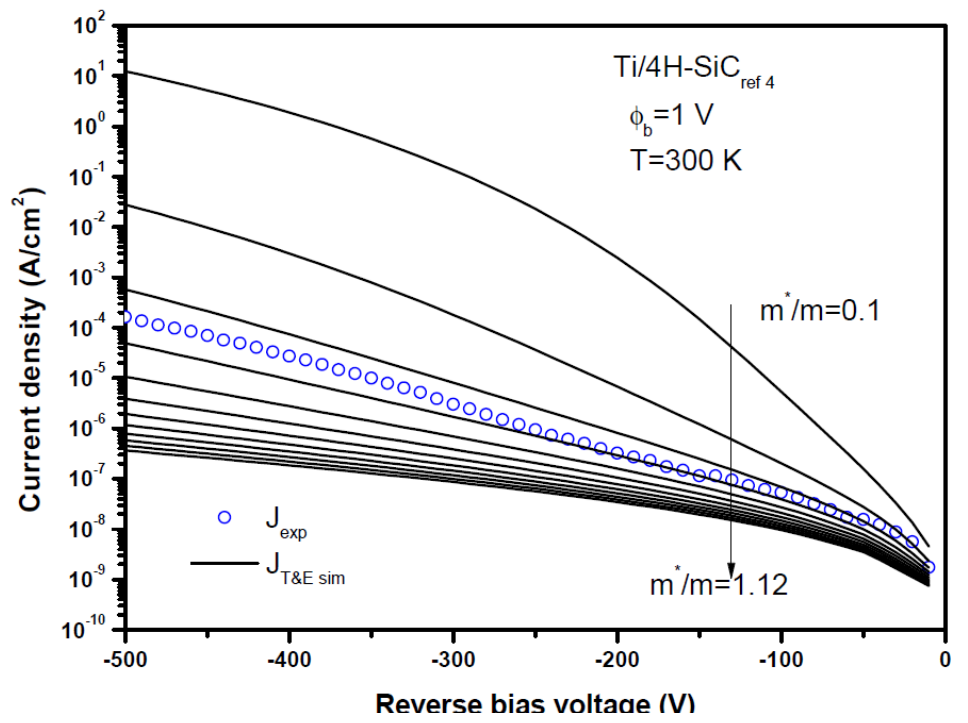

Fig. 2: Current Density Calculated with Tsu-Esaki's Expression as A Function of the Reverse Bias Voltage at Various Effective Mass. with $\phi_{b}=1 \mathrm{~V}$, $\mathrm{T}=300 \mathrm{~K}$.

This result has been mentioned by Furno [4]. However, in this study we have found that the current is decreased when the effective mass is increased because the transmission coefficient is decreased with increasing the effective mass. This behavior is in contradiction with the Furno's results [4]. We explain that by the transfer matrix method used by Furno. In effect, several authors have noted numerical problems in applying this method for the computation of wave functions. These problems are due to the multiplication of matrices with exponentially growing and decaying states. For thick barriers, this leads to rounding errors which eventually exceed the amplitude of the wave function itself [26]. We note here, that, in the case of the forward bias voltage Crowell and Sze [27] found the same behavior as we have obtained. Assuming no reverse bias-dependence of Schottky barrier height and for determining the best values of $\left(\phi_{b}, m^{*}\right)$ for the fit of the experimental data we search the minimum of the sum of the squares of the relative differences between the measured and the ideal values for various values $\left(\phi_{b}, m^{*}\right)$ as shown in Fig. 3. The extracted values of barrier height and effective mass $\left(\phi_{b}, m^{*}\right)$ for $\mathrm{Ti} / 4 \mathrm{H}-\mathrm{SiC}_{\text {ref } 4} \mathrm{SD}$ are $1 \mathrm{~V}$ and $0.345 m$, respectively. The extracted values $\left(\phi_{b}, m^{*}\right)$ for some diodes are collected in Table 2. As shown in Table 2 a significant difference between the extracted values of the effective mass of $4 \mathrm{H}-\mathrm{SiC}$ material for different diodes is observed.

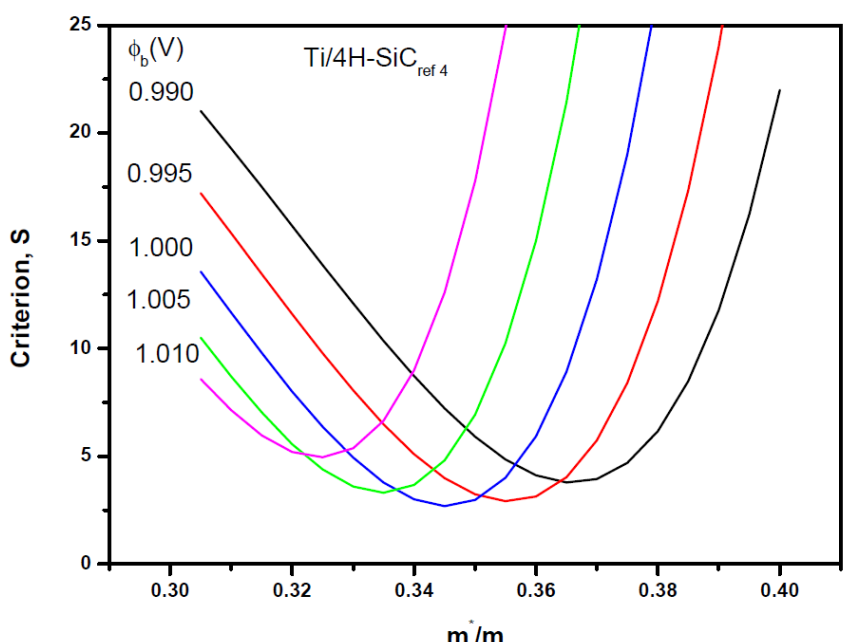

Fig. 3: Minimizing of the Sum of the Quadratic Relative Error (S) as A Function of the Effective Mass at Various Schottky Barrier Heights for the Reverse $I-V$ Data of the $\mathrm{Ti} / 4 \mathrm{H}-\mathrm{SiC}_{\mathrm{ref} 4} \mathrm{SD}$.

Table 2: The Fitting Parameters $\phi_{b}$ and $m^{*}$ Extracted From the Reverse $I$ - $V$ Characteristics for Some Diodes at Room Temperature. The Extracted Values are obtained by Using Tsu-Esaki's Expression (Eq. (10)) with the Criterion, S, Given by the Eq. (9). 


\begin{tabular}{llll}
\hline Type & $\mathrm{N}_{\mathrm{D}}\left(\mathrm{cm}^{-3}\right)$ & $\phi_{b}(\mathrm{~V})$ & $\mathrm{m}^{*} / \mathrm{m}$ \\
\hline $\mathrm{Ti} / 4 \mathrm{H}-\mathrm{SiC}_{\text {ref } 5}$ & $1.6 \times 10^{16}$ & 0.705 & 0.460 \\
$\mathrm{Ti} / 4 \mathrm{H}-\mathrm{SiC}_{\text {ref 18 }}$ & $7 \times 10^{15}$ & 0.840 & 0.435 \\
$\mathrm{Ti}(\mathrm{Ni}) / 4 \mathrm{H}-\mathrm{SiC}_{\text {ref } 6}$ & $3 \times 10^{15}$ & 0.900 & 0.200 \\
$\mathrm{Ni} / 4 \mathrm{H}-\mathrm{SiC}_{\text {ref 22 }}$ & $2 \times 10^{15}$ & 0.985 & 0.050 \\
\hline
\end{tabular}

Fig. 4 shows a comparison between reverse current densities obtained by Tsu-Esaki model calculated with the extracted values reported in Table 2 and the experimental data for several diodes. It can be seen from this figure that the experimental $\ln (\mathrm{I})-\mathrm{V}$ plots and simulated $\ln (\mathrm{I})-\mathrm{V}$ plots do not coincide over the entire bias ranges except in the case of the $\mathrm{Ti}(\mathrm{Ni}) / 4 \mathrm{H}-\mathrm{SiC}_{\text {ref }} 6 \mathrm{SD}$, which exhibits an almost total coincidence. The difference in the nature of the experimental $\ln (\mathrm{I})-\mathrm{V}$ plots and the difference in the effective mass for the same material (4H-SiC) supports the dependence of $\phi_{b}$ on the bias voltage. Furthermore, the experimental curves have different curvatures which differ from each other. The existing of many different curvatures predicts that the variation of the $\mathrm{SBH}$ with bias voltage is not the same for all diodes. Moreover, the coincidence of the experimental and calculated curves for the $\mathrm{Ti}(\mathrm{Ni}) / 4 \mathrm{H}-\mathrm{SiC}$ ref $6 \mathrm{SD}$ predicts no important variation in the barrier height with the bias voltage and the effective mass $\left(m^{*}=0.2 m\right)$ obtained (see Table 2) in this case is equal to the theoretical value.

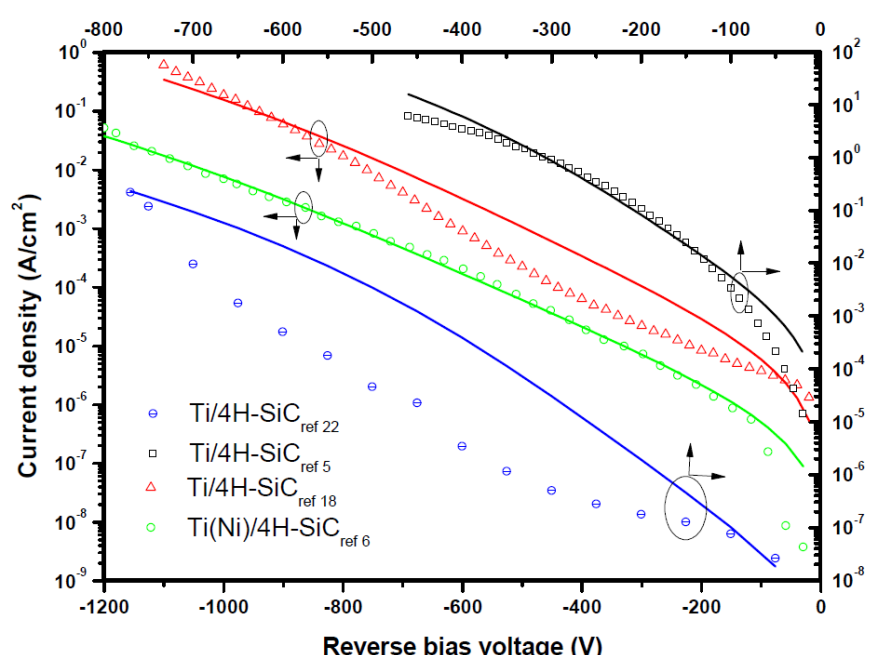

Fig. 4: Experimental and Fitted Reverse $I-V$ Plots for Some Diodes at Room Temperature. Experimental Data are from the References as Reported in Table 1.

For the reasons discussed above, we assume in the following section reverse bias-dependence of Schottky barrier height and we use the previously extraction method discussed in the section 2. Fig. 5 shows the typical dependence of the extracted value of the barrier height on reverse bias using the Tsu-Esaki model (Eq. (10)) for Ti/4H-SiC ${ }_{\text {ref }} 18 \mathrm{SD}_{\text {, at }}$ room temperature with and without the Schottky barrier lowering (SBL) effect. A dependence of $\phi_{b}$ on the reverse bias voltage is clearly observed. Increasing the reverse bias voltage, the barrier height $\phi_{b}$ first increases then starts to decrease again; it exhibits a maximum value of Schottky barrier height. The reverse bias-dependence of Schottky barrier height is independent of the Schottky barrier lowering (SBL) effect as shown in Fig. 5. This behavior is in contradiction with the Schottky effect theory which predicts a decrease in the barrier height when the reverse bias voltage increases. One possible explanation is that other effects occur at the interface in addition to the Schottky barrier lowering. These effects must be strongly dependent on the reverse applied bias than the Schottky barrier lowering effect. 


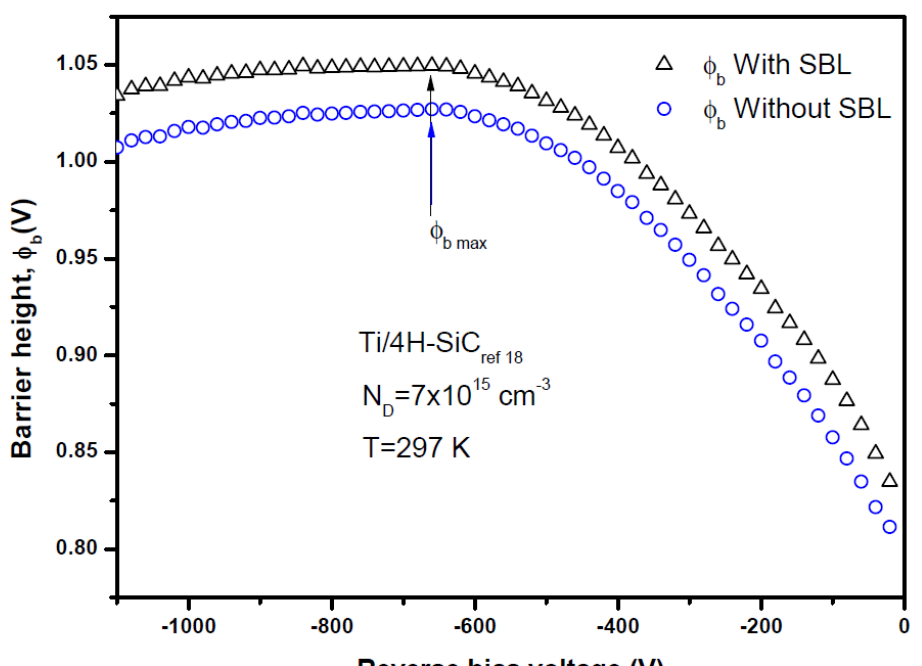

Fig. 5: Schottky Barrier Height as A Function of the Reverse Bias Voltage for Ti/4H-Sic ref $_{18}$ Schottky Diode with and Without Schottky Barrier Lowering Effect. The Values of $\phi_{b}$ Are Extracted By the Tsu-Esaki Model.

The doping concentration dependence of barrier height is depicted in Fig. 6. Fig. 6a shows the barrier height as a function of the reverse bias for $\mathrm{Ti} / 4 \mathrm{H}-\mathrm{SiC}_{\mathrm{ref}}{ }_{23} \mathrm{SDs}$ at different doping concentrations (reverse $I-V$ data are from the same work [23]). While Fig. 6b shows the barrier height as a function of the reverse bias for Ni/4H-SiC SDs at various doping concentrations (reverse $I-V$ data are from several works [5], [6], [21], and [22]). From these figures, we can see two important observations:

First, we can see that the barrier height tends to increase with an increase in the donor concentration, while, several authors [28-31] observed that the barrier height decreases with increasing donor concentration in the case of the forward bias, where the thermionic model is used. Hudait [28] explained this reduction by the barrier height lowering for thermionic field-emission theory, Horváth [29] attributed it to the effect of the interfacial layer and interface states, Noh [30] of his part, attributed this reduction to the Schottky barrier lowering, while, Syrkin [31] explained it by the image force barrier lowering and the value of the surface energy level and the density of surface states.

Second, the barrier height tends to increase with increasing the reverse bias voltage at the high donor concentration (case of Ti/4H-SiC ${ }_{\text {ref } 23}$ and $\mathrm{Ni} / 4 \mathrm{H}-\mathrm{SiC}_{\text {ref } 5} \mathrm{SDs}$ with $N_{D}=7.4 \times 10^{15} \mathrm{~cm}^{-3}$, and $N_{D}=1.6 \times 10^{16} \mathrm{~cm}^{-3}$ respectively), while at the low donor concentration the barrier height tends to decrease with increasing the reverse bias voltage (case of $\mathrm{Ni} / 4 \mathrm{H}$ $\mathrm{SiC}_{22}$ with $N_{D}=2 \times 10^{15} \mathrm{~cm}^{-3}$ ). At intermediate doping concentration we can see an intermediate phase which characterized by no significant variation in the barrier height over the entire bias range, as was expected above (case of $\mathrm{Ti}(\mathrm{Ni}) / 4 \mathrm{H}-\mathrm{SiC}_{\text {ref } 6} \mathrm{SD}$ with $N_{D}=3 \times 10^{15} \mathrm{~cm}^{-3}$, and Ti/4H-SiC ${ }_{\text {ref } 23} \mathrm{SD}$ with $N_{D}=2 \times 10^{15} \mathrm{~cm}^{-3}$ ). The increase in doping concentration leads to a moving of the maximum value of the barrier height from the low reverse bias to the high reverse bias. The value of the barrier height depends on the metal nature as shown in Fig. 7. As shown in this figure the barrier height of the $\mathrm{Ni} / 4 \mathrm{H}-\mathrm{SiC}_{\text {ref }} 5 \mathrm{SD}$ is higher than $\mathrm{Ti} / 4 \mathrm{H}-\mathrm{SiC}_{\text {ref }} 5 \mathrm{SD}$ for the same doping concentration because the metal work function of $\mathrm{Ni}(5.35 \mathrm{eV})$ is higher than $\mathrm{Ti}(4.33 \mathrm{eV})$. It is also seen from Fig. 6 and Fig. 7 that the barrier heights tend to collect at the lower applied bias for the same type of Schottky contact metal where the doping concentration dependence of the barrier height is weak. This result is in close agreement with the doping dependence of the barrier height obtained from forward bias conditions at slightly doping concentration [31].
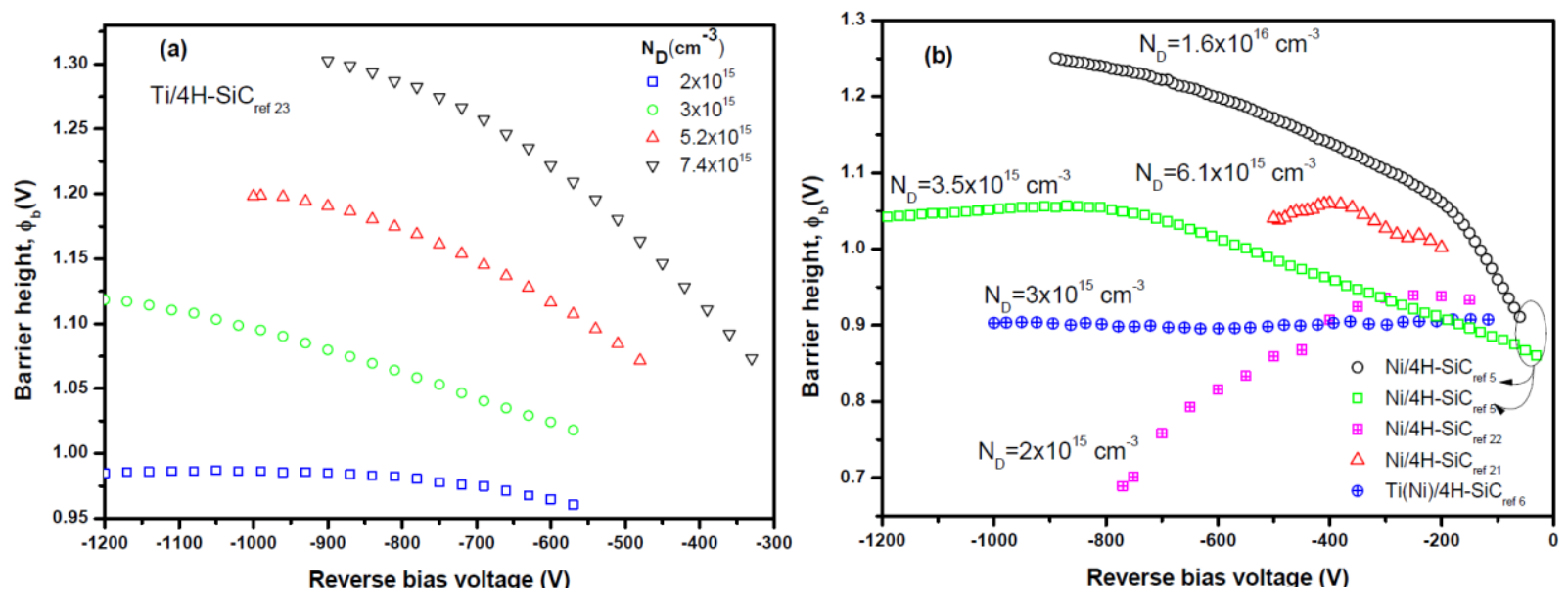

Fig. 6: Schottky Barrier Height as a Function of the Reverse Bias Voltage with Different Donor Concentrations at Room Temperature. (A) For Ti/4H$\mathrm{SiC}_{\text {ref } 23} \mathrm{SDs}$, (B) For Several Metal/4H-SiC ${ }_{\text {ref }}$ SDs. 


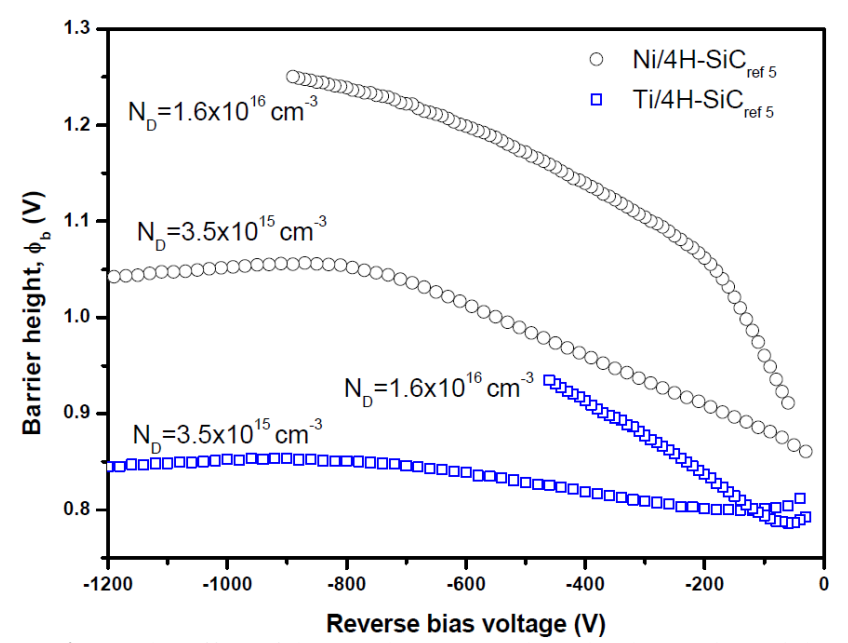

Fig. 7: The Effect of the Metal Nature on the Schottky Barrier Height.

Fig. 8 shows the comparison between the $\phi_{b}$ extracted from the Tsu-Esaki model (Eq. (10)) and the Padovani-Stratton model (Eq. (11) and Eq. (12)). The transition between field and thermionic field emission occurs at approximately -240 $\mathrm{V}$ for $\mathrm{Ni} / 4 \mathrm{H}-\mathrm{SiC}_{\text {ref } 5} \mathrm{SD}$, and $-560 \mathrm{~V}$ for $\mathrm{Ti} / 4 \mathrm{H}-\mathrm{SiC}_{\text {ref } 18} \mathrm{SD}$. It can be clearly seen from this figure that the values of $\phi_{b}$ extracted from the Padovani-Stratton model are close to the values extracted from the Tsu-Esaki model, particularly in the case of thermionic field emission. The little discrepancy between them is due to the fact that the Padovani-Stratton formulas do not include any image force lowering of the barrier and they were derived by considering only the first two terms of the Taylor expansion.

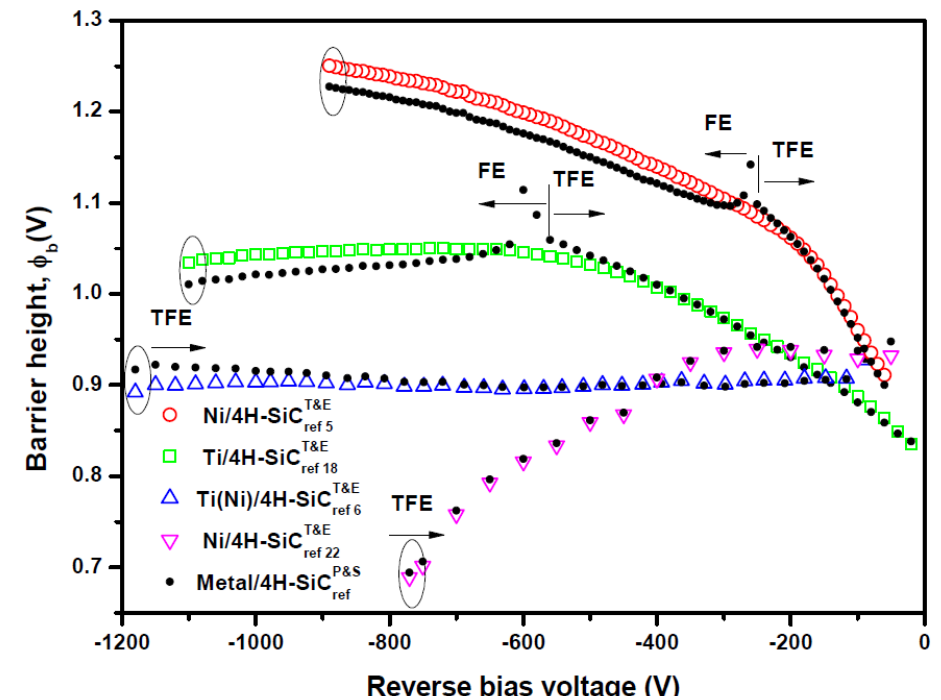

Fig. 8: Comparison between the Values of Schottky Barrier Heights Extracted by Tsu-Esaki's Model (with SBL) and the Values Extracted by Padovani-Stratton Model.

Fig. 9 shows the plots of the extracted barrier height as a function of reverse applied voltage for metal/4H-SiC SDs at different temperatures and doping levels. The increase in temperature leads to an increase in the barrier height, especially, at lower voltages than at higher voltages. As a consequence, the reverse bias dependence is changed and the barrier height tends to decrease with increasing reverse bias in particular at lower doping concentrations (see Fig. 9a, Fig.9b, Fig. 9c and Fig.9d) and the maximum value of the barrier height moves from the high reverse bias to the low reverse bias as shown in Fig. 9e. A reduction in the barrier height value is observed when moving from the forward to the reverse characteristics.

Fig. 10 shows the temperature dependence of the barrier height for metal/4H-SiC SDs, under investigation at different reverse bias. As can be seen from Fig. 10, the experimental barrier height increases linearly with increasing temperature and the slopes are higher for the low bias than for the high bias; the slope is about $1.49 \times 10^{-3} \mathrm{~V} / \mathrm{K}$ at $-500 \mathrm{~V}$ and $1.15 \times 10^{-3} \mathrm{~V} / \mathrm{K}$ at $-1200 \mathrm{~V}$ for $\mathrm{Ti}(\mathrm{Ni}) / 4 \mathrm{H}-\mathrm{SiC}_{\mathrm{ref}}$. This increase in barrier height with increasing temperature is the same behavior at the forward bias, where the thermionic model is used. The authors attributed this temperature dependence to the barrier height inhomogeneities prevailing at the metal-semiconductor interface [19], [32]-[34].

The Schottky barrier height on $\mathrm{SiC}$ is, in general, determined by both the metal work function and the interface states [35]. According to all dependences of barrier height obtained above we can explain them by the combination between 
the effects of the interfacial layer present in intimate metal/4H-SiC contacts and the surface states density on the metalsemiconductor interface. These both effects have a contrary contribution. The interface layer reduces the barrier height by an amount that depends on the applied bias (increase with increasing applied bias), while the interface states increase the barrier height by an amount that depends on the applied bias, doping concentration and temperature (increase with increasing applied bias, temperature and doping concentration). The total barrier height can be written as

$\phi_{b}=\phi_{b}^{\text {th }}\left(\phi_{m}, \chi\right)-\Delta \phi_{b}^{\text {int }-l a y}(V)+\Delta \phi_{b}^{\text {int }- \text { stat }}\left(V, N_{D}, T\right)$

Where $\phi_{b}^{t h}\left(\phi_{m}, \chi\right)$ is the ideal barrier height defined by the difference between the metal work function $\left(\phi_{m}\right)$, and the electron affinity of the semiconductor $(\chi)$.The amount $\Delta \phi_{b}^{\text {int-lay }}(V)$ is due to the polarization of the interfacial layer between the semiconductor and the metal where the dipole layer will orient toward the metal surface. This type of polarization depends on the proprieties of the layer (dielectric permittivity) and the electric field (applied bias). Under external influences such as temperature or electric field (applied voltage) the neutral states (donor interface traps below the neutral level, $\phi_{0}$ ) can be lost their electrons and can be ionized with a positive charge. Consequently, it appears a dipole between the interfacial states (positive charge) on the semiconductor and the metal surface (negative charge) and this dipole will orient toward the semiconductor surface. The result of this effect is the amount $\Delta \phi_{b}^{\text {int }-s t a t}\left(V, N_{D}, T\right)$.

The interface states increase with increasing doping concentration which leads to the increase in the dipole, and hence increase in the barrier height. At high temperature, in particular, at low doping concentration the amount $\Delta \phi_{b}^{\text {int-stat }}\left(V, N_{D}, T\right)$ becomes weak dependent on the applied bias because the majority of interface states can be ionized by the effect of temperature, so, the effect of the interfacial layer which depends on applied bias becomes dominant compared with the effect of the interface states and thus the barrier height tends to decrease with increasing reverse bias.
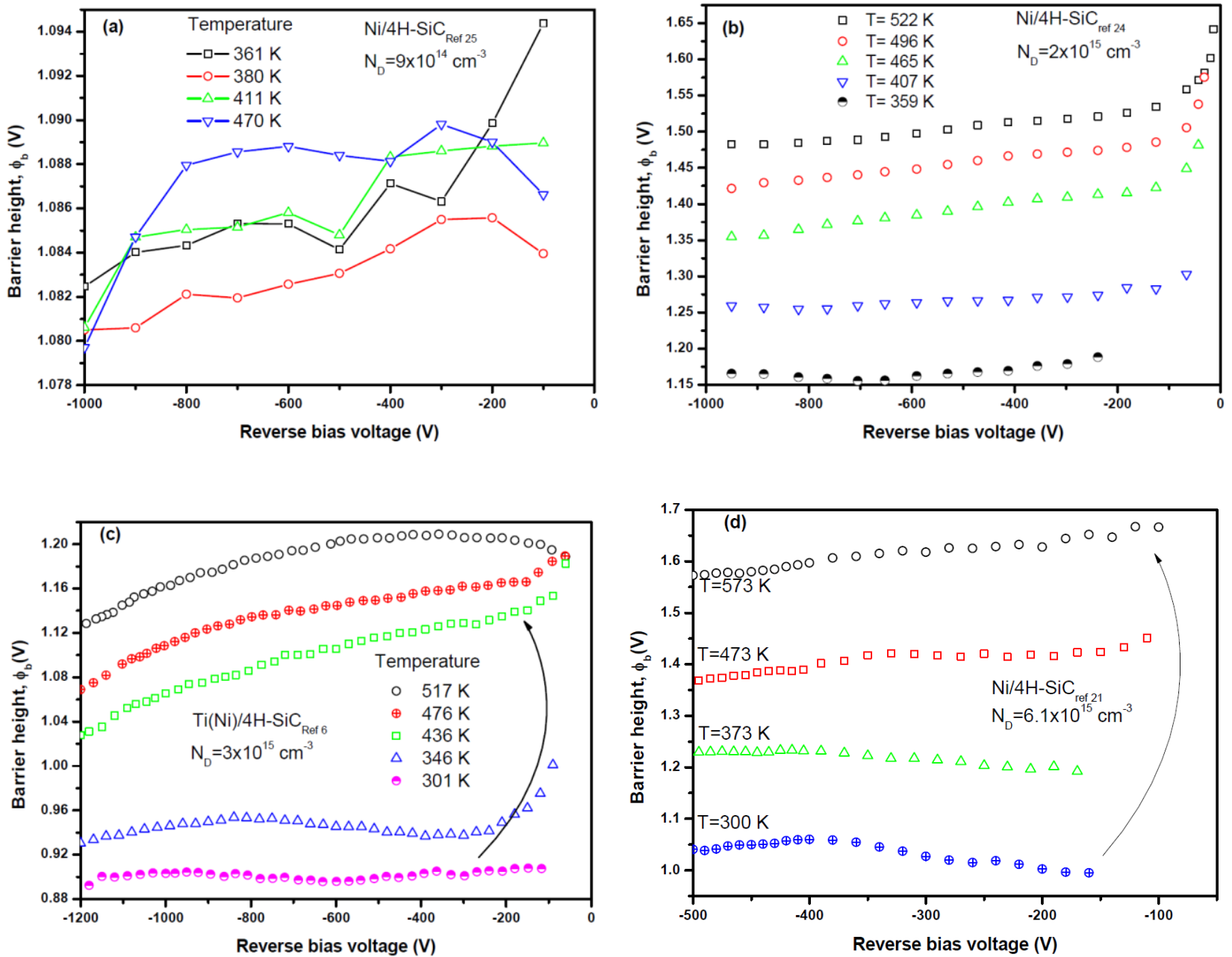

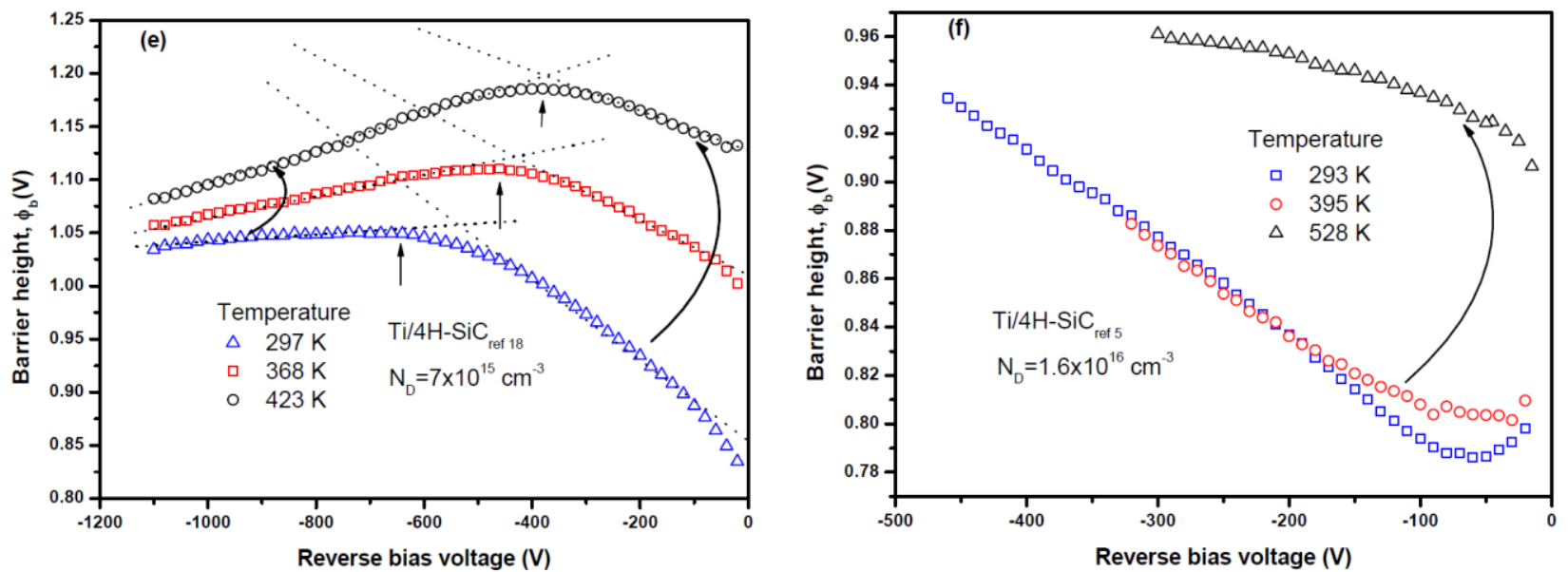

Fig. 9: Schottky Barrier Height as a Function of the Reverse Bias Voltage at Different Temperatures and Different Doping Concentration for Several Metal/4H-SiC ref SDs.

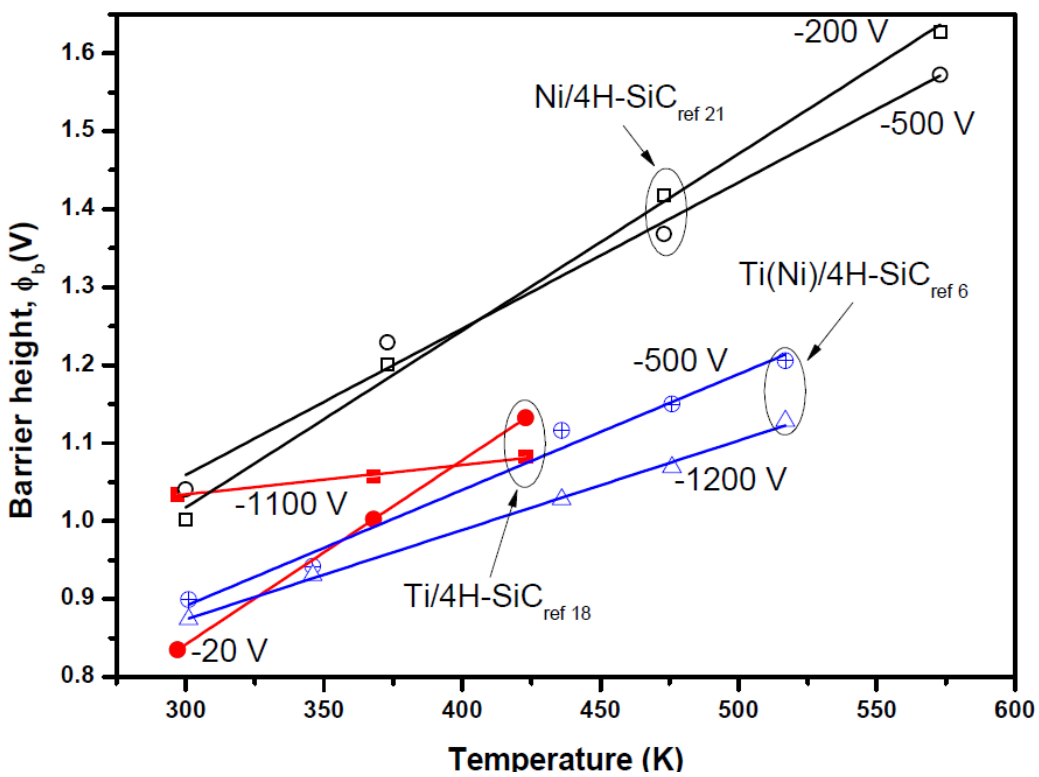

Fig. 10: The Temperature Dependence of the Barrier Height for Several Metal/4H-SiC $\mathrm{C}_{\text {ref }}$ Schottky Diodes at Different Reverse Bias.

\section{Conclusion}

The tunneling modeling is used for extracting the Schottky barrier height from reverse $I-V$ characteristics. The Schottky barrier height extracted from reverse $I-V$ characteristic on silicon carbide exhibits dependences with the reverse applied bias, temperature and doping concentration. At room temperature, the Schottky barrier height increases with increasing the reverse applied bias in the case of high doping concentration and decreases in the case of low doping concentration. This behavior is in contradiction with the behavior predicted by the effect of the image force. That means the existing of other effects which occur at the barrier and depend on the applied bias. The Schottky barrier height increases with increasing temperature and doping concentration. These dependences are due to the combination of the effects of the interfacial layer and interface states. The values of the extracted Schottky barrier height using the Padovani-Stratton model are close to the values of the Schottky barrier height obtained from the Tsu-Esaki model, in particular for thermionic-emission.

\section{References}

[1] A. Mahajan, and B. J. Skromme, "Design and optimization of junction termination extension (JTE) for 4H-SiC high voltage Schottky diodes", Solid-State Electron., Vol. 49, (2005), pp. 945-955. http://dx.doi.org/10.1016/j.sse.2005.03.020.

[2] H. Matsunami, "Current SiC technology for power electronic devices beyond Si, Microelectronic Engineering, Vol. 83, (2006), pp. 2-4. http://dx.doi.org/10.1016/j.mee.2005.10.012.

[3] J. Crofton, and S. Sriram, "Reverse Leakage Current Calculations for SiC Schottky Contacts", IEEE Trans.Electron. Devices, Vol. 43, (1996), pp. 2305-2307. http://dx.doi.org/10.1109/16.544427.

[4] M. Furno, F. Bonani, and G. Ghione, "Transfer matrix method modelling of inhomogeneous Schottky barrier diodes on silicon carbide", Solid-State Electron., Vol. 51, (2007), pp. 466-474. http://dx.doi.org/10.1016/j.sse.2007.01.028. 
[5] K. J. Schoen, J. M. Woodall, J. A. Cooper Jr, and M. R. Melloch, "Design Considerations and Experimental Analysis of High-Voltage SiC Schottky Barrier Rectifiers", IEEE Trans.Electron. Devices, Vol. 45, (1998), pp. 1595-1604. http://dx.doi.org/10.1109/16.701494.

[6] K. Vassilevski, I. Nikitina, P. Bhatnagar, A. Horsfall, N. Wright, A. G. O'Neill, M. Uren, K. Hilton, A. Munday, A. Hydes, and C. M. Johnson, "High temperature operation of silicon carbide Schottky diodes with recoverable avalanche breakdown", Mater. Sci. Forum, 527529, (2006), pp. 931-934. http://dx.doi.org/10.4028/www.scientific.net/MSF.527-529.931.

[7] L. Zheng, R. P. Joshi, and C. Fazi, "Effects of barrier height fluctuations and electron tunneling on the reverse characteristics of $6 \mathrm{H}-\mathrm{SiC}$ Schottky contacts", J. Appl. Phys., Vol. 85, (1999), pp. 3701-3707. http://dx.doi.org/10.1063/1.369735.

[8] D. Blasciuc-Dimitriu, A. B. Horsfall, N. G. Wright, C. M. Johnson, K. V. Vassilevski, and A. G. O'Neill, "Quantum modelling of I-V characteristics for 4H-SiC Schottky barrier diodes", Semicond.Sci.Technol., Vol. 20, (2005), pp. 10-15. http://dx.doi.org/10.1088/0268$1242 / 20 / 1 / 002$.

[9] S. Oyama, T. Hashizume, and H. Hasegawa, "Mechanism of Current Leakage through Metal/n-GaN interfaces", Appl.Surf. Scie., Vol. 190, (2002), pp. 322-325. http://dx.doi.org/10.1016/S0169-4332 (01)00902-3.

[10] E. J. Miller, E. T. Yu, P. Waltereit, and J. S. Speck, "Analysis of reverse-bias leakage current mechanisms in GaN grown by molecular-beam epitaxy", Appl. Phys. Lett., Vol. 84, (2004), pp. 535-537. http://dx.doi.org/10.1063/1.1644029.

[11] E. H. Rhoderick, "Metal-semiconductor contacts", IEE PROC., Vol. 126, (1982), pp. 1-14.

[12] R. Tsu, and L. Esaki, Tunneling in finite superlattice, Appl.phys.lett., Vol. 22, (1973), pp. 562-564. http://dx.doi.org/10.1063/1.1654509.

[13] F. A. Padovani, and R. Stratton, "Field and thermionic-field emission in Schottky barriers", Solid-State Electron., Vol. 9, (1966), pp. 695-707. http://dx.doi.org/10.1016/0038-1101 (66)90097-9.

[14] C. R. Crowell, "The Richardson constant for thermionic emission in Schottky barrier diodes", Solid-State Electron., Vol. 8, (1965), pp. 395399. http://dx.doi.org/10.1016/0038-1101 (65)90116-4.

[15] J. Eriksson, N. Rorsman, and H. Zirath, "4H-Silicon Carbide Schottky Barrier Diodes for Microwave Applications", IEEE Trans.Microwave Theory Technol., Vol. 51, (2003), pp. 796-804. http://dx.doi.org/10.1109/TMTT.2003.808610.

[16] A. Latreche, and Z. Ouennoughi, "Modified Airy function method modeling of tunnelling current for Schottky barrier diodes on silicon carbide", Semicond. Sci. Technol., Vol. 28, (2013), pp. 105003: 1-8

[17] J. Osvald, and E. Dobročka, "Generalized approach to the parameter extraction from I-V characteristics of Schottky diodes", Semicond. Sci. Technol., Vol. 11, (1996), pp. 1198-1202. http://dx.doi.org/10.1088/0268-1242/11/8/014.

[18] A. Itoh, and H. Matsunami, "Analysis of Schottky Barrier Heights of Metal/SiC Contacts and Its Possible Application to High-Voltage Rectifying Devices", phys. stat. sol. (a), Vol. 162, (1997), pp. 389-408.

[19] F. Roccaforte, F. La Via, V. Raineri, R. Pierobon, and E. Zanoni, "Richardson's constant in inhomogeneous silicon carbide Schottky contacts", J. Appl. Phys., Vol. 93, (2003), pp. 9137-9144. http://dx.doi.org/10.1063/1.1573750.

[20] W. Götz, A. Schöner, G. Pensl, W. Suttrop, W. J. Choyke, R. Stein, and S. Leibenzeder, "Nitrogen donors in 4H silicon carbide", J. Appl. Phys., Vol. 73, (1993), pp. 3332-3338. http://dx.doi.org/10.1063/1.352983.

[21] V. Saxena, J. Nong (Jim) Su, and A. J. Steckl, "High-Voltage Ni- and Pt-SiC Schottky Diodes Utilizing Metal Field Plate Termination", IEEE Trans.Electron. Devices, Vol. 46, (1999), pp. 456-464. http://dx.doi.org/10.1109/16.748862.

[22] S. Nigam, J. Kim, B. Luo, F. Ren, G. Y. Chung, S. J. Pearton, J. R. Williams, K. Shenai, and P. Neudeck, "Effect of contact geometry on 4H$\mathrm{SiC}$ rectifiers with junction termination extension", Solid-State Electron., Vol. 47, (2003), pp. 57-60. http://dx.doi.org/10.1016/S0038-1101 (02)00273-3.

[23] K. Ohtsuka, Y. Matsuno, Y. Hase, H. Sugimoto, K. Fujihira, Y. Tarui, M. Imaizumi, T. Takami, and T. Ozeki, "Influence of pinning trap in Ti/4H-SiC Schottky barrier diode", Mat. Sci. semicon. Proc., Vol. 6, (2003), pp. 359-362.

[24] P. A. Ivanov, I. V. Grekhov, A. S. Potapov, T. P. Samsonova, N. D. Il'inskaya, O. I. Kon'kov, and O. Yu Serebrennikova, "Excess Leakage Currents in High-Voltage 4H-SiC Schottky Diodes", Semiconductors, Vol. 44, (2010), pp. 653-656. http://dx.doi.org/10.1134/S1063782610050180.

[25] P. A. Ivanov, I. V. Grekhov, O. I Kon'kov, A. S. Potapov, T. P. Samsonova, and T. V. Semenov, "I-V Characteristics of High-Voltage 4H$\mathrm{SiC}$ Diodes with a 1.1-eV Schottky Barrier", Semiconductors, Vol. 45, (2011), pp. $1374-1377$. http://dx.doi.org/10.1134/S1063782611100095.

[26] A. Gehring, and S. Selberherr, "Modeling of Tunneling Current and Gate Dielectric Reliability for Nonvolatile Memory Devices", IEEE Trans.Device Mater. Reliab., Vol. 4, (2004), pp. 306-319. http://dx.doi.org/10.1109/TDMR.2004.836727.

[27] C. R. Crowell, and S. M. Sze, "Mechanical Reflection of Electrons at Metal- Semiconductor Barriers: Electron Transport in SemiconductorMetal-Semiconductor Structures", J.Appl.phys., Vol. 37, (1966), pp. 2683-2689. http://dx.doi.org/10.1063/1.1782103.

[28] M. K. Hudait, and S. B. Krupanidhi, "Doping dependence of the barrier height and ideality factor of Au/n-GaAs Schottky diodes at low temperatures", Physica B, Vol. 307, (2001), pp. 125-137. http://dx.doi.org/10.1016/S0921-4526 (01)00631-7.

[29] Zs J. Horváth, I. Gyúró, M. Németh-Sallay, and P. Tüttö, "Near-interface concentration reduction in n-type Au/Cr-GaAs Schottky contacts", Vacuum, Vol. 40, (1990), pp. 201-203. http://dx.doi.org/10.1016/0042-207X (90)90156-S.

[30] S. K. Noh, and P. Bhattacharya, "Determination of intrinsic barrier height in the Au/n-GaN contact system", Appl. Phys. Lett., Vol. 78, (2001), pp. 3642-3644. http://dx.doi.org/10.1063/1.1377848.

[31] A. L. Syrkin, J. M. Bluet, G. Bastide, T. Bretagnon, A. A. Lebedev, M. G. Rastegaeva, N. S. Savkina, and V. E. Chelnokov, "Surface barrier height in metal-SiC structures of 6H, 4H and 3C polytype", Mat. Sci.Eng. B, Vol. 46, (1997), pp. 236-239. http://dx.doi.org/10.1016/S09215107 (96)01978-2.

[32] M. E. Aydın, N. Yıldırım, and A. Türüt, "Temperature-dependent behavior of Ni/4H-nSiC Schottky contacts", J. Appl. Phys., Vol. 102, (2007) 04370, pp.1-7.

[33] A. Latreche, Z. Ouennoughi, A. Sellai, R. Weiss, and H. Ryssel, "Electrical characteristics of Mo/4H-SiC Schottky diodes having ionimplanted guard rings: temperature and implant-dose dependence", Semicond. Sci. Technol., Vol. 26, (2011) 085003, pp.1-9.

[34] S. Toumi, A. Ferhat-Hamida, L. Boussouar, A. Sellai, Z. Ouennoughi, and H. Ryssel, "Gaussian distribution of inhomogeneous barrier height in tungsten/4H-SiC (000-1) Schottky diodes", Microelectron. Eng., Vol. 86, (2009), pp. 303-309. http://dx.doi.org/10.1016/j.mee.2008.10.015.

[35] S. M. Sze, and K. Ng. Kwok, "Physics of Semiconductor Devices", 3rd edition, Wiley, New York, (2007). 